\title{
Computed Tomography Image: Guided Needle Biopsy in the Diagnosis of Lung Malignant Tumors under Artificial Intelligence Algorithm
}

\author{
Deping Chen $\left(\mathbb{D},{ }^{1}\right.$ Meng Wang $\left(\mathbb{D},{ }^{2}\right.$ Weiping $\mathrm{Li}(\mathbb{D}),{ }^{1}$ Xin $\mathrm{Li}\left(\mathbb{D},{ }^{1}\right.$ Haiwen Hou ${ }^{\mathbb{D}},{ }^{1}$ \\ and Hui Shan $\mathbb{D}^{1}$ \\ ${ }^{1}$ Imaging Medicine Center, Chenzhou First People' Hospital, Chenzhou 423000, Hunan, China \\ ${ }^{2}$ Imaging and Nuclear Medicine, The First Affiliated Hospital of Zhengzhou University, Zhengzhou 450000, Henan, China \\ Correspondence should be addressed to Hui Shan; 2016216127@smail.jsut.edu.cn
}

Received 14 October 2021; Revised 19 December 2021; Accepted 23 December 2021; Published 11 February 2022

Academic Editor: M Pallikonda Rajasekaran

Copyright (C) 2022 Deping Chen et al. This is an open access article distributed under the Creative Commons Attribution License, which permits unrestricted use, distribution, and reproduction in any medium, provided the original work is properly cited.

\begin{abstract}
The computed tomography (CT) image-guided needle biopsy was applied in the diagnosis of lung malignant tumors based on artificial intelligence (AI) algorithm under convolutional neural network (CNN) to explore the effect of artificial intelligence algorithms segmentation in needle biopsy surgery and to guide the diagnosis of lung malignant tumors. The subjects of the study were 100 patients with lung malignant tumors admitted to the hospital. The cases were diagnosed as lung cancers by CT, and they were divided into two groups with 50 people in each group. Among them, 50 people in the control group did not use algorithms for guidance and 50 people in the experimental group used algorithms for guidance. The 50 patients who received needle biopsy without guidance of any algorithm were included in the control group. The results showed that the average coincidence rate of automatic segmentation and manual segmentation by the artificial intelligence algorithm was $97.46 \%$, and the average false positive rate (FPR) and false negative rate (FNR) were $0.07 \%$ and $0.08 \%$, respectively. The segmentation time of the algorithm group was $12.5 \mathrm{~s}$, which was significantly shorter than the $36.11 \mathrm{~s}$ of the control group, and the segmentation speed was significantly faster. The positive rate of pathology in the control group was $78 \%$, and the pathological positive rate of the algorithm group was $80 \%$. The difference in the pathological positive rate between the two groups was $2 \%$, and the detection effect of the pathological positive rate in the algorithm group was close to that in the control group, which was of positive significance in pathological detection. The satisfaction rate of patients in the algorithm group with the detection effect was $88 \%$, and that of the patients in the control group was $94 \%$. The difference in the detection satisfaction rate between the two groups was $6 \%$. The number of patients with pneumothorax in the algorithm group was 22 , the number of patients with bleeding was 21 , and the number of patients with infection was 2 . The number of patients with pneumothorax in the control group was 17, the number of patients with bleeding was 19, and the number of patients with infection was 3 . The patients had a higher probability of pneumothorax and bleeding and a lower probability of infection; there was no significant difference in the incidence of complications between the two groups of patients. In summary, the intelligent algorithm was effective and feasible in segmenting lesions, and the accuracy of segmentation could meet the clinical needs, which can be used as a reference for surgery. CT images based on artificial intelligence algorithms were a good way to guide the needle biopsy in the diagnosis of lung malignant tumors, improving the accuracy and sensitivity of the diagnosis of lung malignant tumors.
\end{abstract}

\section{Introduction}

Lung cancer is one of the most common malignant tumors, and the mortality rate is very high [1]. When the case is confirmed, the diagnosis of lung cancer is very important. There are many types of lung cancer. Tumorous lesions of lung tissue are the main symptoms. Many systemic diseases can also cause lung tissue lesions, and it is very important to clarify the cause of the lesions [2]. In 1883, Thakur obtained bacteriological data of patients with pneumonia through percutaneous puncture of a brooch [3]. The lung puncture device has evolved from a puncture needle diagnosis to the current coaxial puncture needle diagnosis of tissue cutting. In 1982, Tsai used a semiautomatic biopsy system invented 
by himself to obtain the patient's lung tissue, which improved the success rate of sampling [4]. The use of medical imaging equipment such as X-ray fluoroscopy, computed tomography (CT), magnetic resonance imaging (MRI) under the guidance of percutaneous biopsy is of great significance. CT scans can clearly show the relationship between lung lesions and surrounding tissue anatomy. Now, CT-guided technology is becoming more mature and realizing widely clinical application $[5,6]$. Clinical practice has found that lung cancer can be judged from the local expansion of the internal blood vessels of the disease, the rich blood flow area, or the necrotic gray area; CT scan is more sensitive to image contrast of tumors, small respiratory tracts, liquefied necrosis areas in tumor blood vessels, sternum, and blood vessels between ribs. In surgery, the use of CT-assisted puncture can avoid damage to blood vessels, airways, and lung tissues, thereby improving the sensitivity and accuracy of the diagnosis of lung neoplastic lesions, while reducing complications and providing a clinical basis for early detection and early cancer treatment [7].

At present, lung biopsy has been widely used in clinical surgery and is one of the important methods for the treatment of lung cancer. Pathological defects of lung tissue are difficult to observe, and percutaneous lung puncture is also a major application of lung cancer surgery. It can also be applied to targeted injection, ablation therapy, seed implantation, and other surgical treatments from the chest skin into the final target point of lung disease $[8,9]$. However, the anatomical structure of the human chest is very complicated. During the operation, the biopsy needle will pass through many important tissues and organs, which will cause great difficulties to the operation. In the biopsy puncture process, medical images need to be guided $[10,11]$.

In recent years, with the development of deep learning technology, deep neural networks have achieved a lot of results in computer vision, language processing, and speech recognition. Convolutional neural network (CNN) shows strong performance capabilities for local visual features, has positive significance in image processing, target detection, and image segmentation, and is one of the representative algorithms of deep learning. It has the ability of representation learning and can classify the input information according to its hierarchical structure. The CNN mimics the biological visual perception mechanism, which can perform supervised learning and unsupervised learning. The convolution kernel parameter sharing in the hidden layer and the sparsity of the connections between the layers enable the $\mathrm{CNN}$ to lattice point with a small amount of calculation. $\mathrm{CNN}$ is the key framework used in this research. It is a combination of artificial neural network (ANN) and deep learning. The weights in the $\mathrm{CNN}$ are trained through a backpropagation algorithm to realize deep learning. In recent years, image recognition technology based on data mining, artificial intelligence (AI), and machine learning has begun to be applied in medical imaging. Machine learning focuses more on the design of algorithms, enabling computers to recognize rules from data and use rules to infer unknown data. As a typical representative of deep network algorithms, CNN is currently widely recognized in the field of image recognition.

The guidance methods are divided into ultrasonic guidance, X-ray guidance, and CT guidance. Ultrasonic guidance has no radiation and can be operated in real time [12]. However, because the lungs are filled with a lot of air, ultrasound imaging is very poor. X-ray fluoroscopy guidance can be performed in real time, and the effect of angiography is good, but the radiation to the patient and the doctor is large, and the body tissue is damaged. At present, the most common operation for biopsy of lung lesions is the use of CT-guided percutaneous lung puncture to help doctors obtain CT images of patients. Clinical operations will use surgical navigation systems such as lung puncture operations [13]. The surgical navigation system is based on CT, MRI, and other image data [14]. The corresponding relationship between the preoperative image space and the intraoperative patient space is established under the spatial positioning device, and the position of the biopsy needle during the operation is tracked and displayed in the image space in real time [15]. The location of the biopsy needle and the location of lung tissue lesions are very clear [16]. Therefore, the navigation system for lung puncture surgery can help doctors perform lung puncture operations to improve the success rate and efficiency of the operation and reduce the harm to patients. In this study, artificial intelligence algorithms were used to propose algorithms suitable for understanding lung cancer images, judging diseased tissues, and guiding the entry of biopsy needles. In this study, the clinical effect of CT image-guided needle biopsy based on AI algorithms in the diagnosis of lung malignant tumors was analyzed, and the accuracy, precision, and material satisfaction rate of CT image-guided needle biopsy using AI algorithms were explored. It was expected to be a guide and reference for the biopsy puncture of patients with lung tumors.

\section{Methods}

2.1. Research Objects. The subjects were 100 patients with lung tumorous lesions admitted to the hospital from June 2019 to June 2021. Among them, 52 were males, and 48 were females. They were 20-53 years, with an average age of $36 \pm 8.27$ years. The cases were all diagnosed as lung cancers by CT, and they were divided into two groups with 50 people in each group, one group used and the other group did not use algorithm guidance (control group).

The inclusion criteria were defined as follows: patients with clinical symptoms of lung cancer according to the diagnostic criteria, patients who were not applicable for bronchoscopy, patients who were no younger than 18 years, and patients without other sudden diseases (such as hypertension, heart disease, diabetes, and hypertension).

The exclusion criteria were defined as follows: patients who were allergic to drugs, patients with other sudden diseases, patients under 18 years of age, patients with poor compliance, patients who could not cooperate with treatment such as breath-holding measures, and patients who could not be followed-up. 
This study had been approved by the medical ethics committee of the hospital, and the family members of the patients included in the study had signed the informed consent forms.

\subsection{The Workflow of Lung Puncture Surgery Navigation.} In the clinical operation of lung puncture in patients with lung cancer, it is necessary to locate the lung puncture site in real time, so the operation is performed directly in the CT room. The positioning and operation of lung puncture for lung cancer patients were mainly divided into four stages, as shown in Figure 1.

As shown in Figure 1, there were four steps. First, the patient was required to perform a CTscan of the lungs before surgery. The feature points of the lung space were registered and visualized in the image space. The patient's chest had to be marked with a scan CT machine, so that it can be accurately positioned. Second, the scanned image was imported into the computer to locate the position, size, shape, and other characteristics of the tumor lesion area of the patient. It should mark the skin entry point and puncture path on the CT image and guide the clinical operation according to the planned path. Third, after the path planning of the preoperative scan image, the image space containing the puncture point was established. Then, it could navigate the intraoperative puncture during clinical operations, guide the surgical path based on preoperative CT images, record the blood vessels and the location of the lesion in the patient's lung space, confirm the correspondence between the specific location and the image location based on the patient's body locator, and obtain the space conversion matrix. Fourth, the key point of operation was to use the space locator to obtain the real-time position of the needle detector. The relationship between the space conversion matrix and the real-time position was obtained to obtain the precise puncture position. The preoperative CT image was displayed in real time as a virtual needle, and the insertion can be adjusted. In addition, the insertion direction and depth of the needle can better adjust the surgical effect.

\subsection{Lung Cancer Image Segmentation Technology Based on} Artificial Intelligence. The concept of quaternion was proposed by British mathematician Hamilton in 1843. The application of quaternion now mainly presents the rotation characteristics of the target, which is used to show the specific spatial position in surgical navigation. The quaternion method takes the coordinates of the marker points obtained in the two coordinate systems as the initial 0 coordinates and calculates the rotation matrix $R$ and the translation matrix $T$. The coordinates need to be modified accordingly, and the two spatial coordinate systems are in one-to-one correspondence.

The principle of quaternion is that four numbers represent three axes and an angle. There are four vectors (as given as follows):

$$
q_{k}=\left(q_{0}, q_{1} \cdot q_{2} \cdot q_{3}\right)^{T} .
$$

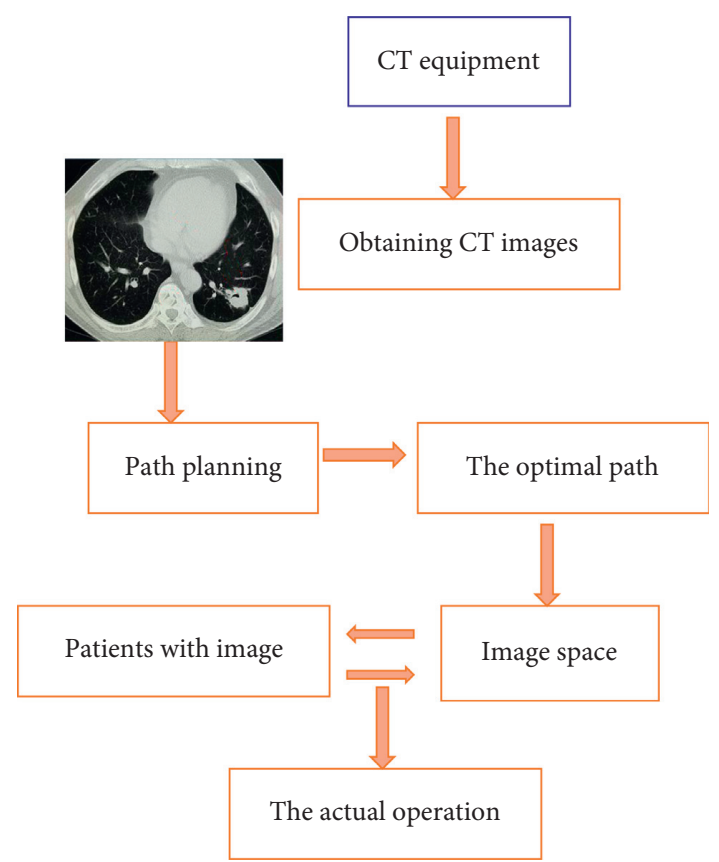

FIGURE 1: The workflow of lung puncture surgery navigation.

These vectors had to meet the following requirements:

$$
q_{0}^{2}+q_{1}^{2}+q_{2}^{2}+q_{3}^{2}=1, \quad q_{0} \geq 0
$$

The set of quaternion was a matrix $R$, as shown in

$$
R=\left[\begin{array}{ccc}
q_{1}^{2}-q_{2}^{2}-q_{3}^{2}+q_{0}^{2} & 2\left(q_{1} q_{2}+q_{3} q_{0}\right) & 2\left(q_{1} q_{3}-q_{2} q_{0}\right) \\
2\left(q_{1} q_{2}-q_{3} q_{0}\right) & -q_{1}^{2}+q_{2}^{2}-q_{3}^{2}+q_{0}^{2} & 2\left(q_{2} q_{3}+q_{1} q_{0}\right) \\
2\left(q_{1} q_{3}+q_{2} q_{1}\right) & 2\left(q_{2} q_{3}-q_{1} q_{0}\right) & -q_{1}^{2}-q_{2}^{2}+q_{3}^{2}+q_{0}^{2}
\end{array}\right] .
$$

The initial axis centers $P_{0}$ and $I_{0}$ were defined as follows:

$$
\begin{gathered}
P_{0}=\frac{1}{n} \sum_{i=1}^{n} P, \\
I_{0}=\frac{1}{n} \sum_{i=1}^{n} L_{i} .
\end{gathered}
$$

$P_{0}$ and $I_{0}$ formed the covariance matrix.

$C=\frac{1}{n} \sum_{i=1}^{n}\left(P_{i}-P_{0}\right)\left(I_{i}-I_{0}\right)^{T}=\frac{1}{n} \sum_{k=1}^{m} P_{i} I_{i}^{T}-P_{0} I_{0}^{T}$.

Covariance finally formed a symmetric matrix, as shown in

$H=\left[\begin{array}{cccc}\operatorname{tr}(C) & C_{12}-C_{21} & C_{20}-C_{02} & C_{01}-C_{10} \\ C_{12}-C_{21} & 2 C_{00}-\operatorname{tr}(C) & C_{01}+C_{10} & C_{02}+C_{20} \\ C_{20}-C_{02} & C_{01}+C_{10} & 2 C_{11}-\operatorname{tr}(C) & C_{12}+C_{21} \\ C_{01}-C_{10} & C_{02}+C_{20} & C_{12}+C_{21} & 2 C_{22}-\operatorname{tr}(C)\end{array}\right]$.

The optimal translation transformation matrix was obtained by $H$.

$$
T=P_{0}-R I_{0}
$$


The transformation matrices $R$ and $H$ adjusted the sets of the two coordinate systems to each other, so as to complete the spatial point registration.

2.4. Evaluation Standard of Experimental Data. Chest CT data had to be evaluated. The patient was scanned using the device from Toshiba Medical, and the scanned image was stored in an uncompressed form (to prevent image distortion during compression). The number of pixels was $512 * 512$, and the layer thickness was $1 \mathrm{~mm}$ and $0.5 \mathrm{~mm}$.

To evaluate the difference in experimental results, the doctor's manual segmentation of the lesion was undertaken as the gold standard to observe the difference between the result of the division and the result of the manual division and calculate the division speed. In addition, the coincidence rate, false positive rate (FPR), and false negative rate (FNR) in terms of the specific performance of the algorithm were calculated.

Coincidence rate referred to the divisor of the gold standard manual segmentation and artificial intelligence automatic segmentation collection, and it could be calculated with the following equation:

$$
C R=\frac{A \cap M}{A \cup M} \text {. }
$$

In the above equation, $\mathrm{CR}$ referred to coincidence rate, $\mathrm{A}$ was the artificial intelligence automatic segmentation collection point, and $M$ represented the gold standard manual segmentation point collection. The larger the coincidence rate, the closer the two methods were. It further showed that the automatic segmentation effect was close to the gold standard and the segmentation effect was better.

The results of manual segmentation of the gold standard were compared with automatic segmentation of artificial intelligence. If the result of artificial intelligence segmentation was more than the result of the gold standard, it was determined as a false positive. The FPR can be expressed as the following equation:

$$
\mathrm{FPR}=\frac{A-A \cap M}{M}
$$

Here, A was the artificial intelligence automatic segmentation collection point, and $M$ represented the gold standard manual segmentation point collection. The larger the value, the more inaccurate points are segmented by artificial intelligence, indicating that the effect is not good.

The results of manual segmentation of the gold standard were compared with automatic segmentation of artificial intelligence. If the result of artificial intelligence segmentation was less than the result of the gold standard, it was determined as a false negative. The FNR can be expressed as the following equation:

$$
\mathrm{FNR}=\frac{M-A \cap M}{M} .
$$

In equation (10), A was the artificial intelligence automatic segmentation collection point, and $M$ represented the gold standard manual segmentation point collection. The larger the value, the more unsegmented lesions of artificial intelligence. It means that many disease points have not been identified, indicating that the effect is not good.

The average number of data layers was 298. The computer configuration was described as follows: random access memory (RAM) was $8 \mathrm{~GB}$, the system was Windows 10 , the processor was Intel Core (TM) i5-5300u, the main frequency was $2.30 \mathrm{GHz}$, and VS2010 was undertaken as the development environment. After the CT scan and before the clinical operation, there is waiting for the patient. At this time, fast and efficient segmentation is required. Therefore, the segmentation time is also a major index for testing automatic segmentation.

2.5. Preoperative Preparation. Before the surgery, it had to perform blood tests and blood coagulation four items, ask if the patient had an acute attack, and perform an electrocardiogram to check patients with severe cardiovascular disease, cerebrovascular disease, chronic obstructive pulmonary disease, etc. If the patient suffered from chronic bronchitis and emphysema (lung puncture was likely to cause severe pneumothorax), it was necessary to inject $60 \mathrm{mg}$ of codeine and $10 \mathrm{mg}$ of diazepam intramuscularly 30 minutes before surgery to suppress cough and reduce pleural reactions. It had to prepare for intraoperative oxygen inhalation measures, check blood oxygen saturation at any time, and sign an informed consent form before the surgery.

2.6. CT Scanning for Positioning. The CT scanning method of the two groups of patients was the same. The patient was required to assist in the posture changes of the supine and prone positions to obtain the optimal scan image. The area from the entrance of the chest cavity to the lower boundary of the lung (costophrenic angle) was the scanning area, and the sensitive organs (eyeball, thyroid, and gonad) were protected by lead clothing. The voltage of the double-row spiral CT machine was $120 \mathrm{kV}$. The elbow vein or wrist vein needed to be supplemented with $1.5 \mathrm{~mL} / \mathrm{kg}$ ioversol injection, which was intravenously injected, and the injection rate was $3.0-4.0 \mathrm{~mL} / \mathrm{s}$.

\subsection{Evaluation Indicators of Surgery Performance.} Satisfaction of obtaining materials: the amount of tissue samples obtained by needle biopsy needed to be greater than $1.0 \mathrm{~cm}$ for histopathological diagnosis.

Pathological positive rate: the result of the tumor lesion area detected by the biopsy was defined as positive, the lung tissue was not inflated, the muscle fiber tissue was diseased, and the bronchial mucosal disease was defined as negative.

Complication rate: pneumothorax refers to the air shadow of the thoracic cavity on the puncture site that can be identified in the chest CT image after the puncture, and it can be identified by the X-ray CT image after the puncture. The detection rate of malignant lung disease and the proportion of malignant lung disease were confirmed pathologically in patients with malignant lung disease. Bleeding during the 
operation not only includes the bleeding from the surgical wound but also counts the hemoptysis after puncturing the lung. The high-density glass shadow around the needle-like passage found in the CT scan after the puncture indicates intrapulmonary bleeding.

2.8. Statistical Analysis. SPSS 20.0 was used for analysis and statistics. Normally distributed measurement data were expressed as mean \pm standard deviation, and comparisons between groups were performed by one-way analysis of variance; general data were by the independent sample $t$-test. A paired-sample $t$-test was used to compare the degree of aneurysm occlusion at different time points in the control group. $P<0.05$ meant the difference was statistically significant.

\section{Results}

3.1. Visual Evaluation and Index Evaluation of Segmentation Results of the Lung Area. The segmentation results and evaluation of the lung area are shown in Figures 2-6. The results showed that the average coincidence rate between the automatic segmentation results of artificial intelligence algorithms and the manual segmentation results was $97.46 \%$, and the average values of FPR and FNR were $0.07 \%$ and $0.08 \%$, respectively. The accuracy rate can meet the clinical needs and can be used as a reference for surgery. The lung segmentation image was more accurate. It can be known from Figures 5 and 6 that the accuracy of AI segmentation was better, the image was clearer, and the resolution effect was better.

3.2. Comparison of Lung Area Segmentation Time. Figures 7 and 8 show the segmentation time comparison of the two methods. The average segmentation time of the algorithm group was $12.5 \mathrm{~s}$, and the average segmentation time of the control group was $36.11 \mathrm{~s}$. The segmentation time of the algorithm group was significantly shorter than the gold standard of the control group, which can greatly reduce the waiting time of patients, and the speed was fast, so it can meet the clinical requirements.

3.3. Comparison of Pathological Positive Rate between Two Groups of Patients. Figure 9 shows the comparison of the pathological positive rate of the two groups of patients, in which $\mathrm{A}$ was the algorithm group and $\mathrm{B}$ was the control group. In the control group, 39 cases were pathologically positive, and the pathological positive rate of the patients was $78 \%$. In the algorithm group, 40 cases were pathologically positive, and the pathologically positive rate of patients was $80 \%$. The difference in the pathological positive rate between the two groups was $2 \%$, indicating that the detection effect of the pathological positive rate of the algorithm group was close to the gold standard of the control group, and the effect was better. Therefore, pathological detection can be better performed.

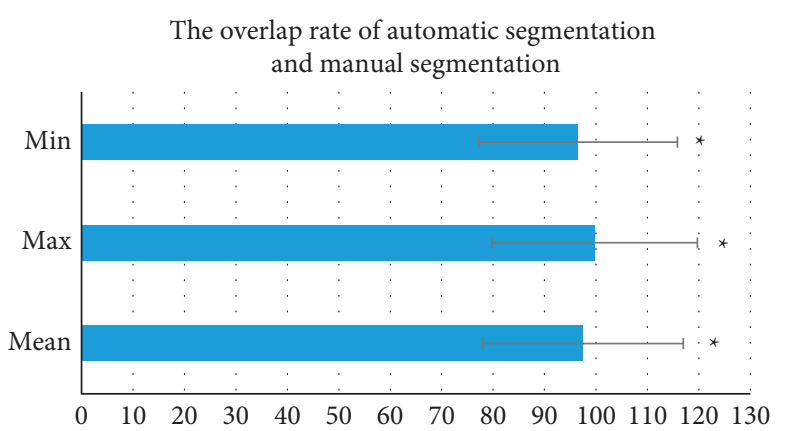

FIGURE 2: The coincidence rate of automatically segmented images and manually segmented images. ${ }^{*}$ The difference was statistically obvious $(P<0.05)$.

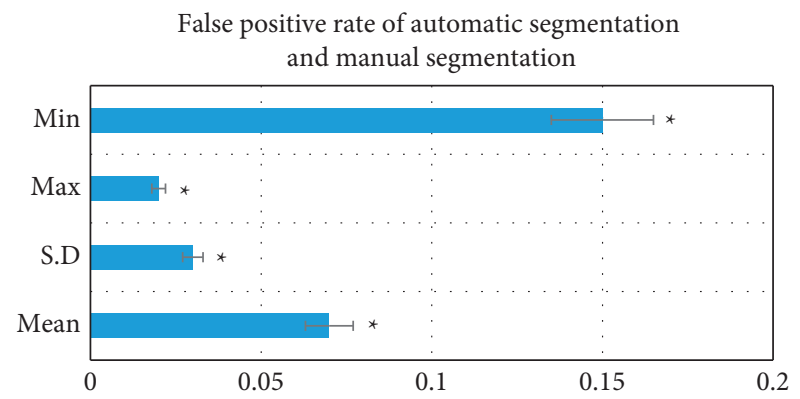

FIGURE 3: FPR of the automatically segmented image and manually segmented image. Note: ${ }^{*}$ the difference was statistically obvious $(P<0.05)$.

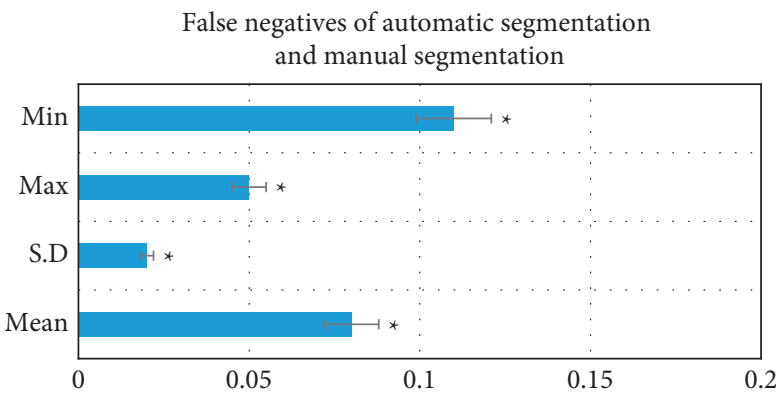

Figure 4: FNR of the automatically segmented image and manually segmented image. Note: ${ }^{*}$ the difference was statistically obvious $(P<0.05)$.

3.4. Comparison of the Detection Satisfaction Rate of the Two Groups of Patients. Figure 10 shows the comparison of the detection satisfaction rate of the two groups of patients, A represented the algorithm group, and $\mathrm{B}$ represented the control group. In the algorithm group, 44 were satisfied with the patients, and 6 were dissatisfied, with a satisfaction rate of $88 \%$. In the control group, 47 patients were satisfied and 3 were dissatisfied, for a patient satisfaction rate of $94 \%$. The difference in the detection satisfaction rate between the two groups was $6 \%$, indicating that the satisfaction degree of the patients in the algorithm group was close to that of the control group, and the patient's recognition was high. 


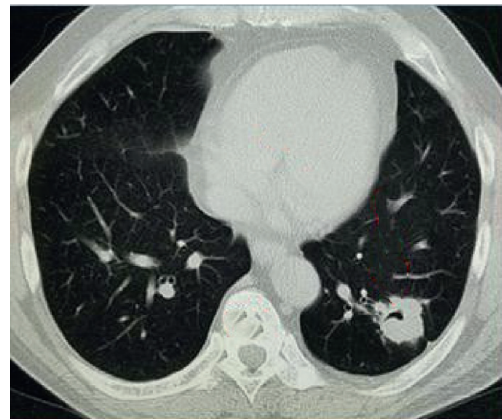

(a)

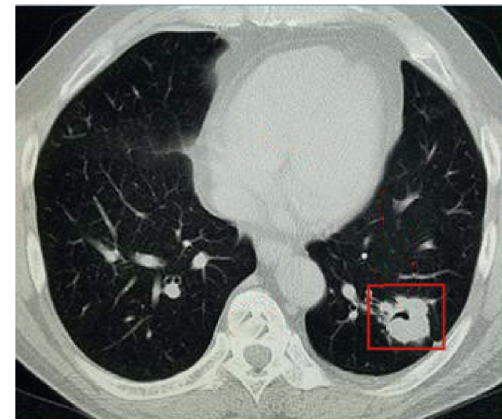

(b)

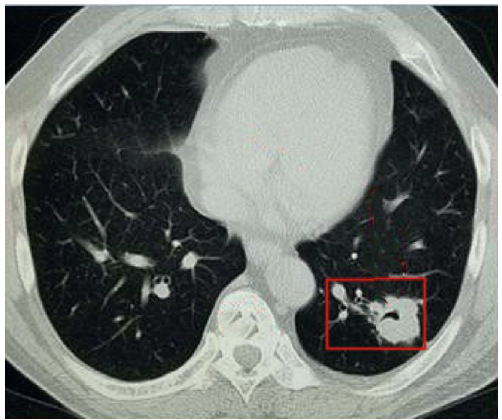

(c)

FIgURE 5: Visual assessments on the accuracy of artificial intelligence segmentation and manual segmentation. Note: (a) original lung cancer medical image, (b) gold standard manual segmentation, and (c) artificial intelligence algorithm segmentation image.

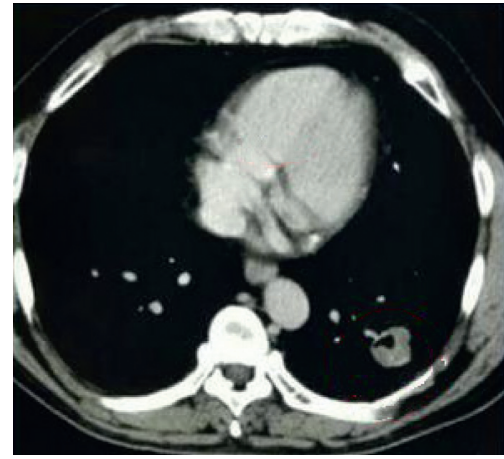

(a)

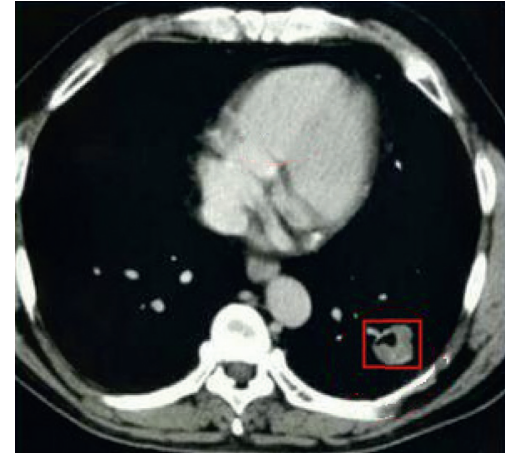

(b)

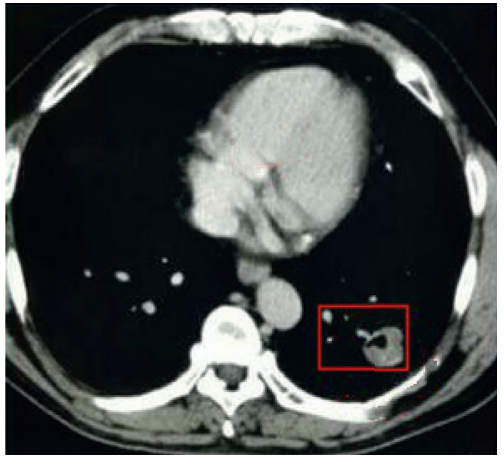

(c)

FIgURE 6: Visual assessments on the accuracy of artificial intelligence segmentation and manual segmentation. Note: (a) original lung cancer medical image, (b) gold standard manual segmentation, and (c) artificial intelligence algorithm segmentation image.

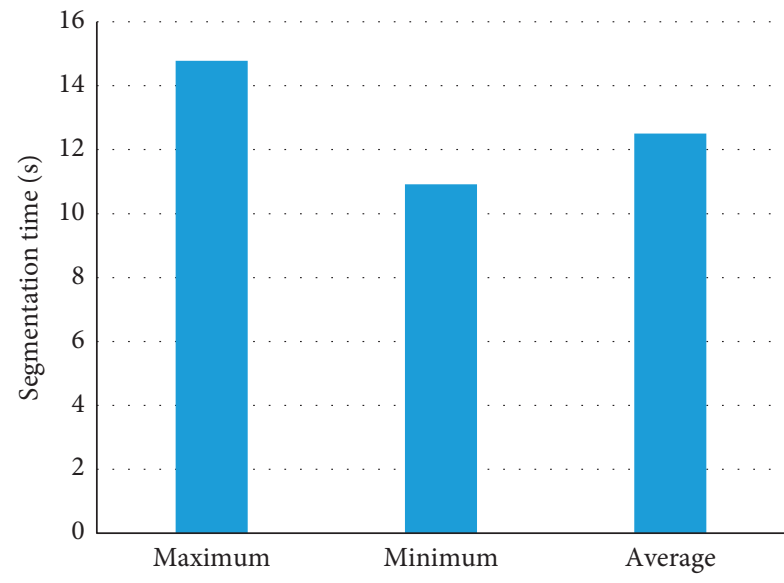

FIgURE 7: Segmentation time of the algorithm group.

\subsection{Comparison of Postoperative Complications.} Figures 11 and 12 show the postoperative complications of the two groups of patients. As illustrated, 22 patients in the algorithm group developed pneumothorax (44\%), 21 patients developed bleeding (42\%), and 2 patients developed an infection (4\%). In the control group, 17 patients had pneumothorax (34\%), 19 patients had bleeding (38\%), and 3 patients had infection (6\%). Comparison of the results of complications revealed that the two groups had a higher probability of pneumothorax and hemorrhage, a lower probability of infection, and no significant difference in the incidence of complications.

\section{Discussion}

Lung cancer is a relatively common lung neoplastic lesion. The common diagnosis method for tumor lesions is to obtain tissue specimens for pathological examination. The usual examination methods include bronchoscopy biopsy, percutaneous lung biopsy, thoracoscopy, open lung biopsy, bronchoscopy detection is more convenient, and there is no trauma, but the diagnosis rate is low. The cost is low, and the patient's inspection process will produce discomfort, causing the patient to refuse to cooperate with the treatment [17]. Thoracoscopy and thoracotomy lung biopsy procedures are various and require intraoperative general anesthesia, the risk is high, and the physical condition of patient does not support anesthesia. In order to obtain tissue specimens, CT-guided percutaneous lung biopsy is a relatively efficient method [18]. In particular, CT scan-guided biopsy as a minimally invasive diagnostic method is widely used in clinical practice [19]. Coaxial needle biopsy can avoid repeated punctures and reduce complications. As a lung tumor identification method, CT emphasizes that scanning coaxial needle biopsy can 


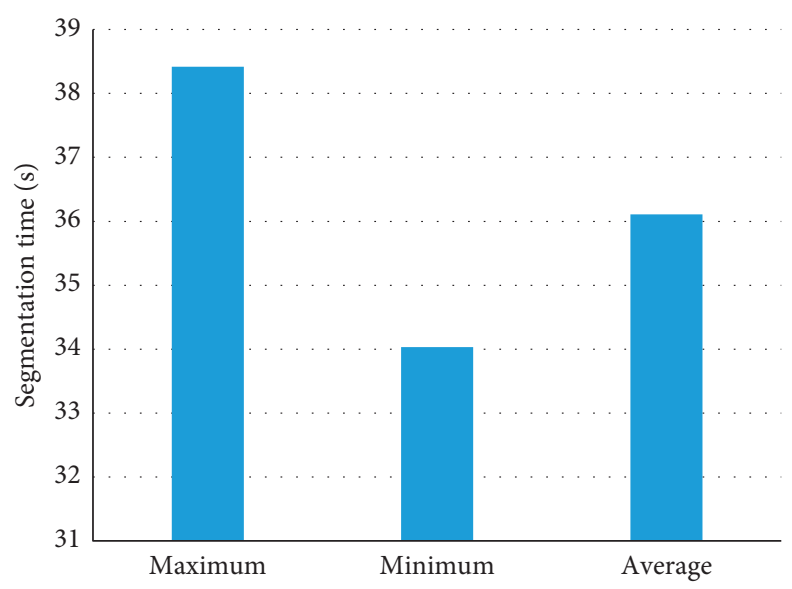

FIGURE 8: Segmentation time of the control group.

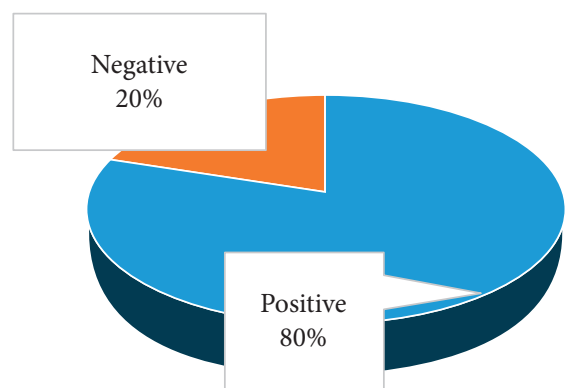

(a)

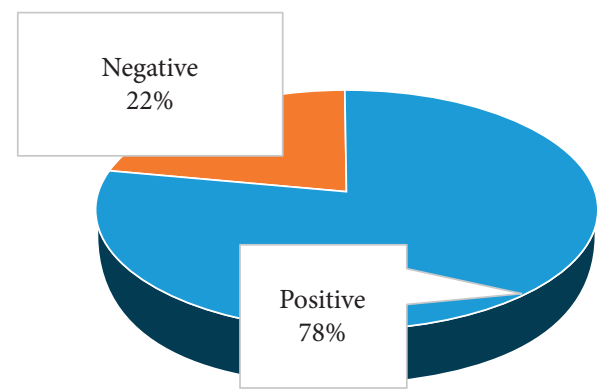

(b)

FIGURE 9: Comparison of the pathological positive rates of the two groups of patients ((a) the rate in the algorithm group; (b) the rate in the control group).

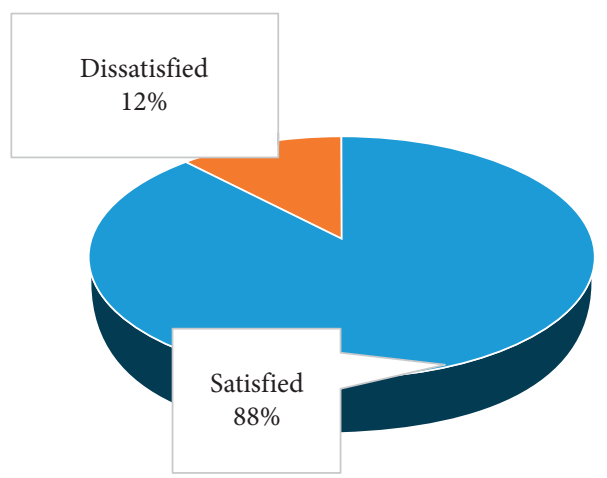

(a)

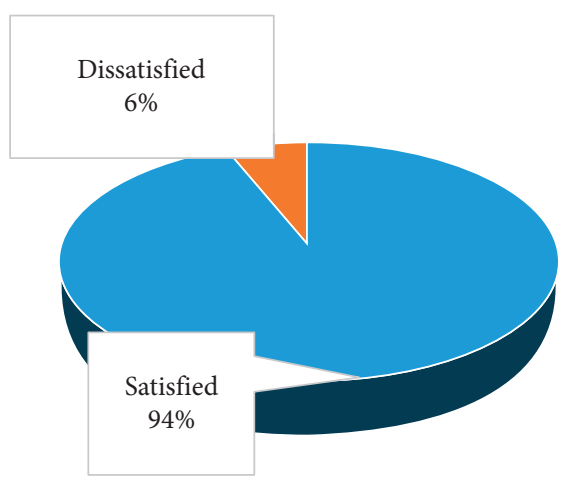

(b)

FIGURE 10: Comparison of the detection satisfaction rate of the two groups of patients ((a) the algorithm group; (b) the control group).

significantly improve the positive detection rate of malignant lesions, which is of great value for the clinical decision of early lung cancer and appropriate treatment options [20]. Through the improvement and development of CT devices, and the improvement of puncture needles, CT-enhanced scanning coaxial needle biopsy can safely and effectively diagnose diseases with fewer complications, especially for lesions that cannot be detected by bronchoscopy. This method is mainly used to determine the puncture point, needle depth, and needle direction of the CT scan lesion location and can accurately obtain pathological examinations and guide clinical treatment specimens [21].

Medical image segmentation is an important field in medical image analysis, and it is also a necessary part of computer-aided diagnosis, monitoring, intervention, and treatment. Medical image segmentation can apply deep learning-based methods to brain, liver, pancreas, prostate, and multiple organ segmentation [22]. Compared with natural images, medical images have many limitations in addition to low resolution, low contrast, and scattered targets, but they have higher requirements for the accuracy 


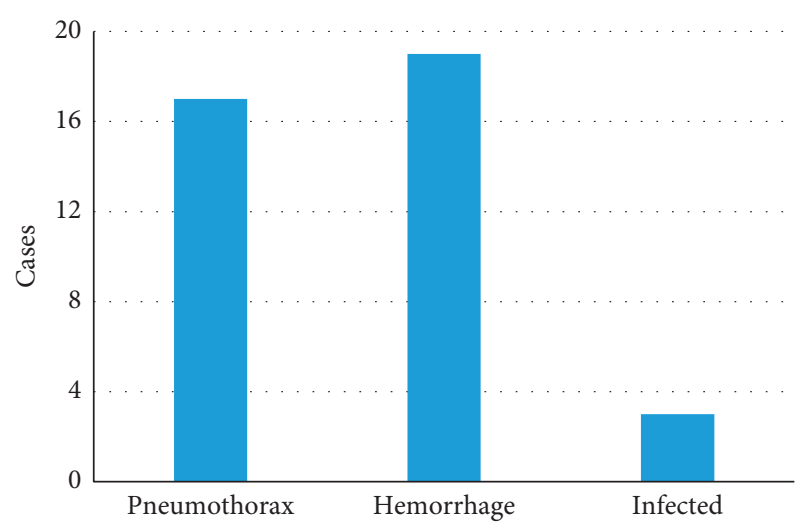

FIgURE 11: Comparison of postoperative complications in the control group.

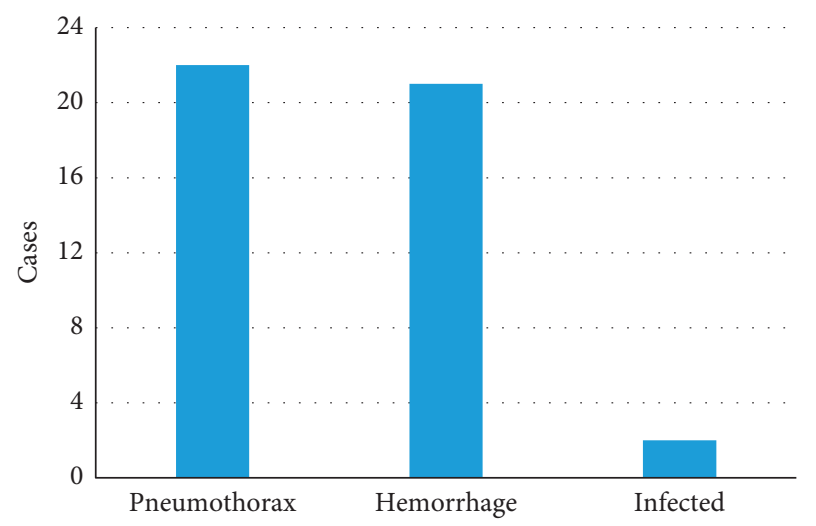

FIgURE 12: Comparison of postoperative complications in the algorithm group.

and stability of the segmentation algorithm results $[23,24]$. The advent of a fully convolutional neural network (FCNN) created a precedent for $\mathrm{CNN}$ for image segmentation. The main method is to change the fully connected layer in the traditional $\mathrm{CNN}$ model to a convolutional layer, use the deconvolution operation to sample on the final output feature map, introduce skip connections to improve the pixel positioning of the rough sampling, and apply the Alex Net, VGG16, and Goog Le Net into the CNN for image classification, becoming a dense prediction network that can achieve image segmentation $[25,26]$. Some studies have found that the application of artificial intelligence algorithms to gamma nail internal fixation under X-ray for the treatment of femoral intertrochanteric fractures has a good guiding effect, good image detail retention, high precision, and positive application effects [27]. The application of CNN to CT image-guided needle biopsy has a positive application value in the diagnosis of lung malignant tumors.

It was found in this study that the average coincidence rate between the automatic segmentation results of artificial intelligence algorithms and the manual segmentation results was $97.46 \%$, and the average values of FPR and FNR were $0.07 \%$ and $0.08 \%$, respectively. The accuracy rate can meet the clinical needs and can be used as a reference for surgery. Based on lung segmentation images, it is also more accurate. The segmentation time of the algorithm group was significantly shorter than that of the control group, the segmentation speed was fast, and the efficiency was high, which can be popularized and applied in clinical practice. In the control group, 39 patients were pathologically positive, and the pathological positive rate was $78 \%$. 40 patients in the algorithm group were pathologically positive, and the pathological positive rate was $80 \%$. The difference in the pathological positive rate between the two groups was $2 \%$, and the detection effect of the pathological positive rate in the algorithm group was close to that in the control group, which was of positive significance in pathological detection. In the algorithm group, 44 patients were satisfied with the detection effect, 6 patients were dissatisfied, and the satisfaction rate was $88 \%$; while in the control group, 47 patients were satisfied, 3 patients were dissatisfied, and the patient satisfaction rate was $94 \%$. The difference in the detection satisfaction rate between the two groups was $6 \%$, and the algorithm group's patient satisfaction effect was close to that of the control group, with better patient satisfaction. In the algorithm group, the incidence of pneumothorax was $44 \%$, the incidence of bleeding was $42 \%$, and the incidence of infection was $4 \%$. In the control group, the incidence of pneumothorax was $34 \%$, the incidence of bleeding was $38 \%$, and the incidence of infection was $6 \%$. The probability of pneumothorax and bleeding was higher, and the probability of infection was lower. There was no significant difference in the incidence of complications between the two groups of patients. The future research direction of this study included processing medical images to analyze the cause of the failure and improve the accuracy of the algorithm, aiming to improve the performance of the algorithm; designing a more efficient and accurate recognition method; applying the algorithm in other medical image processing, analysis, and recognition research to realize an efficient network remote intelligent fracture diagnosis system.

\section{Conclusion}

The CT image-guided needle biopsy based on artificial intelligence algorithms was applied in the diagnosis of lung malignant tumors. The manual classification results on lung tissue lesions of doctors were undertaken as the gold standard, and the algorithm segmentation approximated the results of manual segmentation, indicating that the algorithm was effective and feasible in segmenting lesions. The results showed that the average coincidence rate between the automatic segmentation results of artificial intelligence algorithms and the manual segmentation results was $97.46 \%$, and the average values of FPR and FNR were $0.07 \%$ and $0.08 \%$, respectively. The accuracy rate could meet the clinical needs and can be used as a reference for surgery. The lung segmentation images were also more accurate. Comparison of the two groups of complications suggested that the algorithm group showed fewer complications and was close to the control group, indicating that CT images based on artificial intelligence algorithms were a good way to guide needle biopsy to diagnose lung malignant tumors. 


\section{Data Availability}

The data used to support the findings of this study are available from the corresponding author upon request.

\section{Conflicts of Interest}

The authors declare no conflicts of interest.

\section{Acknowledgments}

This work was supported by the Science and Technology Development Plan Project of Chenzhou Science and Technology Bureau (No. zdyf201834).

\section{References}

[1] H. M. Pascoe, H. C. Knipe, D. Pascoe, and S. B. Heinze, "The many faces of lung adenocarcinoma: a pictorial essay," Journal of Medical Imaging and Radiation Oncology, vol. 62, no. 5, pp. 654-661, 2018.

[2] J. Brioulet, A. David, C. Sagan, L. Cellerin, E. Frampas, and O. Morla, "Percutaneous CT-guided lung biopsy for the diagnosis of persistent pulmonary consolidation," Diagnostic and Interventional Imaging, vol. 101, no. 11, pp. 727-732, 2020.

[3] L. Succony, D. Rassl, A. Barker, F. McCaughan, and R. Rintoul, "Adenocarcinoma spectrum lesions of the lung: detection, pathology and treatment strategies," Cancer Treatment Reviews, vol. 99, Article ID 102237, 2021.

[4] P.-C. Tsai, Y.-C. Yeh, P.-K. Hsu, C.-K. Chen, T.-Y. Chou, and Y.-C. Wu, "CT-guided Core biopsy for peripheral subsolid pulmonary nodules to predict predominant histological and aggressive subtypes of lung adenocarcinoma," Annals of Surgical Oncology, vol. 27, no. 11, pp. 4405-4412, 2020.

[5] R. Y. Brzezinski, I. Vigiser, I. Fomin, L. Israeli, S. ShenharTsarfaty, and A. Bar-Shai, "The yield of immediate post lung biopsy CT in predicting iatrogenic pneumothorax," BMC Pulmonary Medicine, vol. 20, no. 1, p. 91, 2020.

[6] N. Becker, E. Motsch, A. Trotter et al., "Lung cancer mortality reduction by LDCT screening-Results from the randomized German LUSI trial," International Journal of Cancer, vol. 146, no. 6, pp. 1503-1513, 2020.

[7] E. Appel, S. Dommaraju, A. Camacho et al., "Dependent lesion positioning at CT-guided lung biopsy to reduce risk of pneumothorax," European Radiology, vol. 30, no. 11, pp. 6369-6375, 2020.

[8] A.-C. Toffart, S. Asfari, A. Mc Leer et al., "Percutaneous CTguided biopsy of lytic bone lesions in patients clinically suspected of lung cancer: diagnostic performances for pathological diagnosis and molecular testing," Lung Cancer, vol. 140, pp. 93-98, 2020.

[9] Y. Onishi, K. Ito, N. Motoi, T. Morita, S.-i. Watanabe, and M. Kusumoto, "Ciliated muconodular papillary tumor of the lung: 18F-FDG PET/CT findings of 15 cases," Annals of Nuclear Medicine, vol. 34, no. 6, pp. 448-452, 2020.

[10] Y. Han, H. J. Kim, K. A. Kong et al., "Diagnosis of small pulmonary lesions by transbronchial lung biopsy with radial endobronchial ultrasound and virtual bronchoscopic navigation versus CT-guided transthoracic needle biopsy: a systematic review and meta-analysis," PLoS One, vol. 13, no. 1, Article ID e0191590, 2018.
[11] T. Leger, N. Jerjir, J. Gregory et al., "Does ipsilateral-dependent positioning during percutaneous lung biopsy decrease the risk of pneumothorax?" American Journal of Roentgenology, vol. 212, no. 2, pp. 461-466, 2019.

[12] W. Yu, Z. An, Z. Wang, W. Lv, and J. Hu, "[CT-guided percutaneous lung puncture for the diagnosis of solid pulmonary nodules: a single-center experience summary]," Zhongguo Fei Ai Za Zhi, vol. 23, no. 6, pp. 414-418, 2020, Chinese.

[13] O. Drumm, E. A. Joyce, C. de Blacam et al., "CT-guided lung biopsy: effect of biopsy-side down position on pneumothorax and chest tube placement," Radiology, vol. 292, no. 1, pp. 190-196, 2019.

[14] G. Fang, N.-C. Cheng, L.-L. Huang, W.-P. Xie, C.-M. Hu, and W. Chen, "The first report of co-existence of pulmonary tuberculosis and lung malignancy in a kidney transplant recipient: a case report and literature review," BMC Infectious Diseases, vol. 21, no. 1, p. 629, 2021.

[15] J. Yoon, Y. J. Suh, K. Han et al., "Utility of CT radiomics for prediction of $\mathrm{PD}-\mathrm{L} 1$ expression in advanced lung adenocarcinomas," Thoracic Cancer, vol. 11, no. 4, pp. 993-1004, 2020.

[16] Q. Li, X. Li, X.-Y. Li, J.-W. Huo, F.-J. Lv, and T.-Y. Luo, "Spectral CT in lung cancer: usefulness of iodine concentration for evaluation of tumor angiogenesis and prognosis," American Journal of Roentgenology, vol. 215, no. 3, pp. 595-602, 2020.

[17] Y. R. Huo, M. V. Chan, A.-R. Habib, I. Lui, and L. Ridley, "Pneumothorax rates in CT-Guided lung biopsies: a comprehensive systematic review and meta-analysis of risk factors," British Journal of Radiology, vol. 93, no. 1108, Article ID 20190866, 2020.

[18] C. Floridi, A. Carnevale, E. M. Fumarola et al., "Percutaneous lung tumor biopsy under CBCT guidance with PETCT fusion imaging: preliminary experience," CardioVascular and Interventional Radiology, vol. 42, no. 11, pp. 1644-1648, 2019.

[19] L. Zeng, H. Liao, F. Ren, Y. Zhang, Q. Wang, and M. Xie, "Pneumothorax induced by computed tomography guided transthoracic needle biopsy: a review for the clinician," International Journal of General Medicine, vol. 14, pp. 10131022, 2021.

[20] S. Hadique, P. Jain, Y. Hadi, A. Baig, and J. E. Parker, "Utility of FDG PET/CT for assessment of lung nodules identified during low dose computed tomography screening," BMC Medical Imaging, vol. 20, no. 1, p. 69, 2020.

[21] L. Dominguez-Konicki, A. R. Karam, M. S. Furman, and D. J. Grand, "CT-guided biopsy of pulmonary nodules $\leq 10 \mathrm{~mm}$ : diagnostic yield based on nodules' lobar and segmental distribution," Clinical Imaging, vol. 66, pp. 7-9, 2020.

[22] M. Hu, Y. Zhong, S. Xie, H. Lv, and Z. Lv, "Fuzzy system based medical image processing for brain disease prediction," Frontiers in Neuroscience, vol. 15, p. 965, 2021.

[23] Z. Yu, S. U. Amin, M. Alhussein, and Z. Lv, "Research on disease prediction based on improved DeepFM and IoMT," IEEE Access, vol. 9, pp. 39043-39054, 2021.

[24] Y. Li, J. Zhao, Z. Lv, and J. Li, "Medical image fusion method by deep learning," International Journal of Cognitive Computing in Engineering, vol. 2, pp. 21-29, 2021.

[25] S. Xie, Z. Yu, and Z. Lv, "Multi-disease prediction based on deep learning: a survey," Computer Modeling in Engineering and Sciences, vol. 128, no. 2, pp. 489-522, 2021. 
[26] Y. Wu, L. Lin, J. Wang, and S. Wu, "Application of semantic segmentation based on convolutional neural network in medical images," Sheng Wu Yi Xue Gong Cheng Xue Za Zhi, vol. 37, no. 3, pp. 533-540, 2020, Chinese.

[27] Y. Wang, X. Wang, L. Jin, and X. Wei, "X-ray film under artificial intelligence algorithm in the evaluation for nursing effect of gamma nail internal fixation in elderly patients with intertrochanteric fracture of femur," Comput Math Methods Med, vol. 2021, Article ID 2562575, 2021. 